\title{
Global Stability of a Stage-Structured Predator-Prey Model with Stochastic Perturbation
}

\author{
Liu Yang and Shouming Zhong \\ School of Mathematics Science, University of Electronic Science and Technology of China, Chengdu 611731, China \\ Correspondence should be addressed to Liu Yang; ylazx@126.com
}

Received 14 October 2013; Accepted 4 January 2014; Published 24 February 2014

Academic Editor: Leonid Shaikhet

Copyright (C) 2014 L. Yang and S. Zhong. This is an open access article distributed under the Creative Commons Attribution License, which permits unrestricted use, distribution, and reproduction in any medium, provided the original work is properly cited.

\begin{abstract}
This paper is concerned with a new predator-prey model with stage structure on prey, in which the immature prey and the mature prey are preyed on by predator. We think that the model is more realistic and interesting than the one in which only the immature prey or the mature prey is consumed by predator. Our work shows that the stochastic model and its corresponding deterministic system have a unique global positive solution and the positive solution is global asymptotic stability for each model. If the positive equilibrium point of the deterministic system is globally stable, then the stochastic model will preserve the nice property provided that the noise is sufficiently small. Results are analyzed with the help of graphical illustrations.
\end{abstract}

\section{Introduction}

Within the past decades, the dynamic behavior between predator and their prey has received considerable interest due to their wide applications in ecology and mathematical ecology. There is a great deal of attention for predator-prey models from many scholars [1-9]. In most of the cases, the study is based on interactions between homogeneous populations. However, in the nature, most of the species must go through two life stages from birth to death. In [6-9], some stage-structured models of population growth consisting of immature and mature individuals were discussed. In particular, [9] considered a predator-prey model with two populations, that is, predator and their food prey. In their model, only prey species is divided into two life stages, the immature prey and the mature prey. And the predators only consume the immature prey species. In [10], Chinese firebellied newt is described as an example, which is unable to prey on the mature Rana chensinensis and can only prey on the immature one. So, they consider the following nonlinear ordinary differential equations:

$$
\frac{d x_{1}}{d t}=\alpha x_{2}-r_{1} x_{1}-\eta x_{1}^{2}-\beta x_{1}-\beta_{1} x_{1} x_{3}
$$

$$
\begin{gathered}
\frac{d x_{2}}{d t}=\beta x_{1}-r_{2} x_{2} ; \\
\frac{d x_{3}}{d t}=-r x_{3}+k \beta_{1} x_{1} x_{3}-\eta_{1} x_{3}^{2} .
\end{gathered}
$$

In [11], the authors have studied the dynamical properties of deterministic model (1) and the stochastic behavior of the corresponding stochastic model (19), which was first introduced by Beretta et al. [12] and Shaikhet [13]. Then, they obtained stochastic stability condition in mean square sense by utilizing Lyapunov function.

On one hand, the predators functional response, that is, the rate of prey consumption by an average predator, is one of the important components which can impact the relationship between predator and prey in population dynamics. There are many functional responses such as Holling type [5], Beddington-DeAngelis type [7], and Watt type [14]. On the other hand, population is inevitably affected by environmental noise in nature $[15,16]$. May [17] also showed that, due to environmental fluctuation, the birth rate, the death rate, competition coefficients, and other parameters usually show random fluctuation to a certain extent 
that should be stochastic. Therefore, many authors have taken stochastic perturbation into deterministic models and shown the effect of environmental variability on population dynamics in mathematical ecology [18-26]. For example, [26] considered the following stochastic stage-structured predator-prey model:

$$
\begin{aligned}
d x_{1}=\left[r x_{2}-\right. & \left.d_{1} x_{1}-a x_{1}^{2}-b x_{1}\right] d t-\sigma_{1} x_{1}\left(x_{1}-x_{1}^{*}\right) d B_{1}(t) \\
d x_{2}= & {\left[b x_{1}-d_{2} x_{2}-a_{11} x_{2}^{2}-\frac{a_{12} x_{2} x_{3}}{1+m x_{2}+n x_{3}}\right] d t } \\
& -\sigma_{2} x_{2}\left(x_{2}-x_{2}^{*}\right) d B_{2}(t) ; \\
d x_{3}= & {\left[-d_{3} x_{3}+\frac{a_{21} x_{1} x_{3}}{1+m x_{2}+n x_{3}}-a_{22} x_{3}^{2}\right] d t } \\
& -\sigma_{3} x_{3}\left(x_{3}-x_{3}^{*}\right) d B_{3}(t) .
\end{aligned}
$$

In this paper, the authors mainly utilize Itô's formula, the theory of stochastic differential equations, and Lyapunov functions to investigate the global stability of the positive equilibrium of model (2).

Motivated by the above works, in this paper, we will consider the following stochastic stage-structured predatorprey model:

$$
\begin{aligned}
d x_{1}= & {\left[r x_{2}-d_{1} x_{1}-a_{11} x_{1}^{2}-b x_{1}-\frac{a_{13} x_{1} x_{3}}{1+m x_{1}+n x_{3}}\right] d t } \\
& -\sigma_{1} x_{1}\left(x_{1}-x_{1}^{*}\right) d B_{1}(t) ; \\
d x_{2}= & {\left[b x_{1}-d_{2} x_{2}-a_{22} x_{2}^{2}-\frac{a_{23} x_{2} x_{3}}{\alpha+\beta x_{2}}\right] d t } \\
& -\sigma_{2} x_{2}\left(x_{2}-x_{2}^{*}\right) d B_{2}(t) ; \\
d x_{3}= & {\left[-d_{3} x_{3}+\frac{k_{1} a_{13} x_{1} x_{3}}{1+m x_{1}+n x_{3}}+\frac{k_{2} a_{23} x_{2} x_{3}}{\alpha+\beta x_{2}}-a_{33} x_{3}^{2}\right] d t } \\
& -\sigma_{3} x_{3}\left(x_{3}-x_{3}^{*}\right) d B_{3}(t),
\end{aligned}
$$

where $x_{1}(t)$ denotes the population density of the immature prey, $x_{2}(t)$ represents the population density of the mature prey, and $x_{3}(t)$ stands for the population size of predator. $B_{i}(t)(i=1,2,3)$ is a standard Brownian motion, which is defined on a complete probability space $(\Omega, \mathscr{F}, P)$ with a filtration $\left\{\mathscr{F}_{t}\right\}_{t \in R_{+}}$satisfying the usual conditions (i.e., right continuous and increasing while $\mathscr{F}_{0}$ contains all $\mathscr{P}_{\text {-null }}$ sets). All parameters involved with the model are positive constants and can be interpreted in more detail: $r$ is the birth rate of immature prey population, $d_{1}, d_{2}$, and $d_{3}$ represent the death rate of immature prey population, mature prey population, and predator population, respectively, $a_{11}, a_{22}$, and $a_{33}$ denote intraspecies competition rate of immature prey population, mature prey population, and predator population, respectively, $b$ represents transformation rate from immature prey population to mature prey population, $a_{i 3}(i=$ $1,2)$ is the rate of predation, $k_{i}(i=1,2)$ is fraction of prey biomass converted into predator biomass, $x_{1} /\left(1+m x_{1}+n x_{3}\right)$ represents Beddington-DeAngelis functional response and $x_{2} /\left(\alpha+\beta x_{2}\right)$ denotes Holling-II functional response, and $\sigma_{i}^{2}(i=1,2,3)$ is the intensity of the noise.

The initial condition of model (3) is any point in the biological meaning region $R_{+}^{3}=\left\{\left(x_{1}, x_{2}, x_{3}\right) \in R^{3} \mid x_{1}>\right.$ $\left.0, x_{2}>0, x_{3}>0\right\}$. $\left(x_{1}^{*}, x_{2}^{*}, x_{3}^{*}\right)$ is a positive equilibrium of model (3) which is the solution of the algebraic equations

$$
\begin{gathered}
r x_{2}-d_{1} x_{1}-a_{11} x_{1}^{2}-b x_{1}-\frac{a_{13} x_{1} x_{3}}{1+m x_{1}+n x_{3}}=0 \\
b x_{1}-d_{2} x_{2}-a_{22} x_{2}^{2}-\frac{a_{23} x_{2} x_{3}}{\alpha+\beta x_{2}}=0 \\
-d_{3} x_{3}+\frac{k_{1} a_{13} x_{1} x_{3}}{1+m x_{1}+n x_{3}}+\frac{k_{2} a_{23} x_{2} x_{3}}{\alpha+\beta x_{2}}-a_{33} x_{3}^{2}=0
\end{gathered}
$$

with initial conditions $x_{i}(0)>0, i=1,2,3$. Noting that if $\sigma_{1}=\sigma_{2}=\sigma_{3}=0$, then model (3) becomes the following corresponding deterministic stage-structured predator-prey systems:

$$
\begin{gathered}
\frac{d x_{1}}{d t}=r x_{2}-d_{1} x_{1}-a_{11} x_{1}^{2}-b x_{1}-\frac{a_{13} x_{1} x_{3}}{1+m x_{1}+n x_{3}} \\
\frac{d x_{2}}{d t}=b x_{1}-d_{2} x_{2}-a_{22} x_{2}^{2}-\frac{a_{23} x_{2} x_{3}}{\alpha+\beta x_{2}} \\
\frac{d x_{3}}{d t}=-d_{3} x_{3}+\frac{k_{1} a_{13} x_{1} x_{3}}{1+m x_{1}+n x_{3}}+\frac{k_{2} a_{23} x_{2} x_{3}}{\alpha+\beta x_{2}}-a_{33} x_{3}^{2} .
\end{gathered}
$$

Therefore, in this paper, we only need to establish the sufficient conditions for global asymptotic stability of system (3). And, in this paper, we will also use Itô's formula, the theory of stochastic differential equations, and Lyapunov functions to study the global stability of the positive equilibrium point of stochastic system (3).

The paper is organized as follows. In Section 2, we study the existence and uniqueness of global positive solution of system (3). In Section 3, sufficient conditions for global asymptotic stability of system (3) are established. Then, we introduce some simulation figures to illustrate the main result in Section 4. In the last section, we give the conclusions.

\section{Existence and Uniqueness of Solution}

In this section, we will show that the solution of system (3) is positive and global. We give the following theorem.

Theorem 1. For any given initial value $\left(x_{1}(0), x_{2}(0), x_{3}(0)\right) \epsilon$ $R_{+}^{3}$, there is a unique solution $\left(x_{1}(t), x_{2}(t), x_{3}(t)\right)$ to (3) on $t \geq$ 0 . Furthermore, with probability one, $R_{+}^{3}$ are positive invariant for (3); that is, for all $t \geq 0,\left(x_{1}(t), x_{2}(t), x_{3}(t)\right) \in R_{+}^{3}$ a.s., if $\left(x_{1}(0), x_{2}(0), x_{3}(0)\right) \in R_{+}^{3}$.

Proof. Since the coefficients of (3) are locally Lipschitz continuous, there is a unique local solution $\left(x_{1}(t), x_{2}(t), x_{3}(t)\right)$ to (3) on $t \in\left[0, \tau_{e}\right)$, where $\tau_{e}$ is the explosion time $[27,28]$. Therefore, to show that the solution is global, we only need 
to show that $\tau_{e}=\infty$ a.s. We use the technique of localization $[29,30]$. Let $k_{0}>0$ be sufficiently large for $x_{i}(0)(i=1,2,3)$ lying within the interval $\left[1 / k_{0}, k_{0}\right]$. Let us define a sequence of stopping time [29] for each integer $k \geq k_{0}$ by

$$
\tau_{k}=\inf \left\{t \in\left[0, \tau_{e}\right): x_{i}(t) \notin\left(\frac{1}{k}, k\right), i=1,2,3\right\} .
$$

The convention here is that the infimum of the empty set is $\infty$. Since $\tau_{k}$ is nondecreasing as $k \rightarrow \infty$, we set $\tau_{\infty}=\lim _{k \rightarrow \infty} \tau_{k}$. Then, $\tau_{\infty} \leq \tau_{e}$ a.s. Now, we will show that $\tau_{\infty}=\infty$ a.s. If the statement is not right, then there exist $T>0$ and $\varepsilon \in(0,1)$ such that $P\left\{\tau_{\infty} \leq T\right\}>\varepsilon$. Thus, by denoting $\Omega_{k}=\left\{\tau_{k} \leq T\right\}$, there exists $k_{1} \geq k_{0}$ such that

$$
P\left(\Omega_{k}\right) \geq \varepsilon \quad \forall k \geq k_{1}
$$

Consider the following function: $V\left(x_{1}, x_{2}, x_{3}\right)=2 \sum_{i=1}^{3}\left(\sqrt{x_{i}}-\right.$ $\left.1-0.5 \ln x_{i}\right)$. It is clear to see that $V \in C^{2}\left(R_{+}^{3}, R_{+}\right)$. If $\left(x_{1}(t), x_{2}(t), x_{3}(t)\right) \in R_{+}^{3}$, by using Itô formula, we get

$$
d V\left(x_{1}, x_{2}, x_{3}\right)=f\left(x_{1}, x_{2}, x_{3}\right) d t-\sum_{i=1}^{3} g_{i}\left(x_{1}, x_{2}, x_{3}\right) d B_{i}(t)
$$

where

$$
\begin{aligned}
f\left(x_{1}, x_{2}, x_{3}\right) \\
=\frac{\left(\sqrt{x_{1}}-1\right)}{x_{1}} \\
+\left[r x_{2}-d_{1} x_{1}-a_{11} x_{1}^{2}-b x_{1}-\frac{a_{13} x_{1} x_{3}}{1+m x_{1}+n x_{3}}\right] \\
+\frac{\sigma_{1}^{2}}{4}\left(-\sqrt{x_{1}}+2\right)\left(x_{1}-x_{1}^{*}\right)^{2} \\
+\frac{\left(\sqrt{x_{2}}-2\right)}{x_{2}}\left[b x_{1}-d_{2} x_{2}-a_{22} x_{2}^{2}-\frac{a_{23} x_{2} x_{3}}{\alpha+\beta x_{2}}\right] \\
+\frac{\sigma_{2}^{2}}{4}\left(-\sqrt{x_{2}}+2\right)\left(x_{2}-x_{2}^{*}\right)^{2}, \\
+\frac{\left(\sqrt{x_{3}}-1\right)}{x_{3}} \\
\times\left[-d_{3} x_{3}+\frac{k_{1} a_{13} x_{1} x_{3}}{1+m x_{1}+n x_{3}}+\frac{k_{2} a_{23} x_{2} x_{3}}{\alpha+\beta x_{2}}-a_{33} x_{3}^{2}\right] \\
+\frac{\sigma_{3}^{2}}{4}\left(-\sqrt{x_{3}}+2\right)\left(x_{3}-x_{3}^{*}\right)^{2} \\
\quad g_{i}\left(x_{1}, x_{2}, x_{3}\right)=\sigma_{i}\left(\sqrt{x_{i}}-1\right)\left(x_{i}-x_{i}^{*}\right) .
\end{aligned}
$$

Since all coefficients of system (2) are positive constants, it is easy to see from (9) that the function $f\left(x_{1}, x_{2}, x_{3}\right)$ is bounded, say by $M$. Thus, since $\left(x_{1}\left(t \wedge \tau_{k}\right), x_{2}\left(t \wedge \tau_{k}\right)\right.$, and $\left.x_{3}\left(t \wedge \tau_{k}\right)\right) \in R_{+}^{3}$ and we consider (9), we have

$$
\begin{aligned}
& \int_{0}^{T \wedge \tau_{k}} d V\left(x_{1}(t), x_{2}(t), x_{3}(t)\right) \\
& \quad \leq \int_{0}^{T \wedge \tau_{k}} M d t-\sum_{i=1}^{3} \int_{0}^{T \wedge \tau_{k}} g_{i}\left(x_{1}, x_{2}, x_{3}\right) d B_{i}(t) .
\end{aligned}
$$

Taking expectations yields

$$
\begin{aligned}
& E V\left(x_{1}\left(T \wedge \tau_{k}\right), x_{2}\left(T \wedge \tau_{k}\right), x_{3}\left(T \wedge \tau_{k}\right)\right) \\
& \quad \leq V\left(x_{1}(0), x_{2}(0), x_{3}(0)\right)+\int_{0}^{T \wedge \tau_{k}} M d t \\
& \quad \leq V\left(x_{1}(0), x_{2}(0), x_{3}(0)\right)+M T .
\end{aligned}
$$

On the other hand, for every $\omega \in \Omega_{k}$, either $x_{1}\left(\tau_{k}, \omega\right)$ or $x_{2}\left(\tau_{k}, \omega\right)$ or $x_{3}\left(\tau_{k}, \omega\right)$ equals either $k$ or $1 / k$. Then,

$$
\begin{aligned}
& V\left(x_{1}\left(T \wedge \tau_{k}, \omega\right), x_{2}\left(T \wedge \tau_{k}, \omega\right), x_{3}\left(T \wedge \tau_{k}, \omega\right)\right) \\
& \quad \geq \min \left\{\sqrt{k}-1-0.5 \ln k, \sqrt{\frac{1}{k}}-1-0.5 \ln \left(\frac{1}{k}\right)\right\} .
\end{aligned}
$$

We therefore get from (7) that

$$
\begin{aligned}
& E V\left(x_{1}\left(t \wedge \tau_{k}\right), x_{2}\left(t \wedge \tau_{k}\right), x_{3}\left(t \wedge \tau_{k}\right)\right) \\
& \quad \geq E\left[1_{\Omega_{k}} V\left(x_{1}\left(t \wedge \tau_{k}\right), x_{2}\left(t \wedge \tau_{k}\right), x_{3}\left(t \wedge \tau_{k}\right)\right)\right] \\
& \quad \geq \varepsilon \min \left\{\sqrt{k}-1-0.5 \ln k, \sqrt{\frac{1}{k}}-1-0.5 \ln \left(\frac{1}{k}\right)\right\},
\end{aligned}
$$

where $1_{\Omega_{k}}$ is the indicator function of $\Omega_{k}$. Then, it follows from (11) that

$$
\begin{aligned}
& V\left(x_{1}(0), x_{2}(0), x_{3}(0)\right)+M T \\
& \quad \geq \varepsilon \min \left\{\sqrt{k}-1-0.5 \ln k, \sqrt{\frac{1}{k}}-1-0.5 \ln \left(\frac{1}{k}\right)\right\} .
\end{aligned}
$$

Letting $k \rightarrow \infty$ leads to the contradiction $\infty>$ $V\left(x_{1}(0), x_{2}(0), x_{3}(0)\right)+M T=\infty$. Therefore, $\tau_{\infty}=\infty$ a.s. Then, $\tau_{e}=\infty$ a.s. and $\left(x_{1}(t), x_{2}(t), x_{3}(t)\right) \in R_{+}^{3}$ a.s. This completes the proof of Theorem 1 .

\section{Globally Asymptotically Stable}

For the sake of convenience, denote

$$
\begin{aligned}
& A=-\frac{x_{1}^{*}}{r}\left[a_{11}-\frac{m a_{13} x_{3}^{*}}{1+m x_{1}^{*}+n x_{3}^{*}}-\frac{\sigma_{1}^{2} x_{1}^{*}}{2}\right], \\
& B=-\frac{x_{2}^{*}}{b}\left[a_{22}-\frac{a_{23} \beta x_{3}^{*}}{\alpha\left(\alpha+\beta x_{2}^{*}\right)}-\frac{\sigma_{2}^{2} x_{2}^{*}}{2}\right], \\
& C=-\frac{x_{1}^{*}\left(1+m x_{1}^{*}\right)}{r k_{1}\left(1+n x_{3}^{*}\right)}\left[a_{33}-\frac{\sigma_{3}^{2} x_{3}^{*}}{2}\right], \\
& D=\frac{a_{23} x_{2}^{*}}{b \alpha}+\frac{k_{2} a_{23} x_{1}^{*}\left(1+m x_{1}^{*}\right)}{r k_{1}\left(1+n x_{3}^{*}\right)\left(\alpha+\beta x_{2}^{*}\right)} .
\end{aligned}
$$


Theorem 2. If

$$
\begin{aligned}
& A<0, \\
& C<0
\end{aligned}
$$

as well as

$$
4 B C-D^{2}>0
$$

Then, the positive equilibrium position $\left(x_{1}^{*}, x_{2}^{*}, x_{3}^{*}\right)$ of model (3) is globally asymptotically stable with probability one; that is, for any positive initial data $\left(x_{1}(0), x_{2}(0), x_{3}(0)\right)$, the solution of system (3) has the property that

$$
\lim _{t \rightarrow \infty} x_{i}(t)=x_{i}^{*} \quad(i=1,2,3)
$$

almost surely.

Proof. From the stability theory of stochastic differential equations, we only need to find a suitable Lyapunov function $V(z)$ satisfying $L V(z) \leq 0$ and the identity holds if and only if $z=z^{*}$ [30], where $z=z(t)$ is the solution of the following stochastic differential equation:

$$
d z(t)=f(t, z(t)) d t+g(t, z(t)) d B(t),
$$

$z^{*}$ is the positive equilibrium position of (19), and

$$
L V(z)=V_{z}(z) f(t, z)+0.5 \operatorname{trace}\left[g^{T}(t, z) V_{z z}(z) g(t, z)\right] .
$$

For $x \in R_{+}^{3}$, define

$$
V(x)=\sum_{i=1}^{3} \frac{c_{i}}{2} \int_{x_{i}^{*}}^{x_{i}} \frac{x_{i}-x_{i}^{*}}{x_{i}} d x_{i}
$$

where

$$
c_{1}=\frac{x_{1}^{*}}{r}, \quad c_{2}=\frac{x_{2}^{*}}{b}, \quad c_{3}=\frac{c_{1}\left(1+m x_{1}^{*}\right)}{k_{1}\left(1+n x_{3}^{*}\right)} .
$$

We can rewrite (3) as follows:

$$
\begin{aligned}
d x_{1}= & \frac{r}{x_{1}^{*}}\left[x_{1}\left(x_{2}-x_{2}^{*}\right)-x_{2}\left(x_{1}-x_{1}^{*}\right)\right] d t \\
& -a_{11} x_{1}\left(x_{1}-x_{1}^{*}\right) d t \\
& -a_{13} x_{1} \frac{\left(1+m x_{1}^{*}\right)\left(x_{3}-x_{3}^{*}\right)-m x_{3}^{*}\left(x_{1}-x_{1}^{*}\right)}{\left(1+m x_{1}+n x_{3}\right)\left(1+m x_{1}^{*}+n x_{3}^{*}\right)} d t \\
& -\sigma_{1} x_{1}\left(x_{1}-x_{1}^{*}\right) d B_{1}(t) ; \\
d x_{2}= & \frac{b}{x_{2}^{*}}\left[x_{2}\left(x_{1}-x_{1}^{*}\right)-x_{1}\left(x_{2}-x_{2}^{*}\right)\right] d t \\
& -a_{22} x_{2}\left(x_{2}-x_{2}^{*}\right) d t \\
& -a_{23} x_{2} \frac{\left(\alpha+\beta x_{2}^{*}\right)\left(x_{3}-x_{3}^{*}\right)-\beta x_{3}^{*}\left(x_{2}-x_{2}^{*}\right)}{\left(\alpha+\beta x_{2}\right)\left(\alpha+\beta x_{2}^{*}\right)} d t \\
& -\sigma_{2} x_{2}\left(x_{2}-x_{2}^{*}\right) d B_{2}(t) ;
\end{aligned}
$$

$$
\begin{aligned}
d x_{3}= & -a_{33} x_{3}\left(x_{3}-x_{3}^{*}\right) d t \\
& +k_{1} a_{13} x_{3} \frac{\left(1+n x_{3}^{*}\right)\left(x_{1}-x_{1}^{*}\right)-n x_{1}^{*}\left(x_{3}-x_{3}^{*}\right)}{\left(1+m x_{1}+n x_{3}\right)\left(1+m x_{1}^{*}+n x_{3}^{*}\right)} d t \\
& +k_{2} a_{23} x_{3} \frac{\alpha\left(x_{2}-x_{2}^{*}\right)}{\left(\alpha+\beta x_{2}\right)\left(\alpha+\beta x_{2}^{*}\right)} d t \\
& -\sigma_{3} x_{3}\left(x_{3}-x_{3}^{*}\right) d B_{3}(t) .
\end{aligned}
$$

Applying Itô formula to model (23), we can get that

$L V(x)$

$$
\begin{aligned}
& =\frac{x_{1}^{*}}{r}\left\{\left(x_{1}-x_{1}^{*}\right) \frac{r}{x_{1}^{*}}\left[\left(x_{2}-x_{2}^{*}\right)-\frac{x_{2}}{x_{1}}\left(x_{1}-x_{1}^{*}\right)\right]\right. \\
& -a_{11}\left(x_{1}-x_{1}^{*}\right)^{2} \\
& -a_{13} \frac{\left(1+m x_{1}^{*}\right)\left(x_{3}-x_{3}^{*}\right)-m x_{3}^{*}\left(x_{1}-x_{1}^{*}\right)}{\left(1+m x_{1}+n x_{3}\right)\left(1+m x_{1}^{*}+n x_{3}^{*}\right)} \\
& \left.\times\left(x_{1}-x_{1}^{*}\right)+\frac{\sigma_{1}^{2} x_{1}^{*}}{2}\left(x_{1}-x_{1}^{*}\right)^{2}\right\} \\
& +\frac{x_{2}^{*}}{b}\left\{\left(x_{2}-x_{2}^{*}\right) \frac{b}{x_{2}^{*}}\left[\left(x_{1}-x_{1}^{*}\right)-\frac{x_{1}}{x_{2}}\left(x_{2}-x_{2}^{*}\right)\right]\right. \\
& -a_{22}\left(x_{2}-x_{2}^{*}\right)^{2} \\
& -a_{23} \frac{\left(\alpha+\beta x_{2}^{*}\right)\left(x_{3}-x_{3}^{*}\right)-\beta x_{3}^{*}\left(x_{2}-x_{2}^{*}\right)}{\left(\alpha+\beta x_{2}\right)\left(\alpha+\beta x_{2}^{*}\right)} \\
& \left.\times\left(x_{2}-x_{2}^{*}\right)+\frac{\sigma_{2}^{2} x_{2}^{*}}{2}\left(x_{2}-x_{2}^{*}\right)\right\} \\
& +\frac{x_{1}^{*}\left(1+m x_{1}^{*}\right)}{r k_{1}\left(1+n x_{3}^{*}\right)} \\
& \times\left\{-a_{33}\left(x_{3}-x_{3}^{*}\right)^{2}+k_{1} a_{13}\right. \\
& \times \frac{\left(1+n x_{3}^{*}\right)\left(x_{1}-x_{1}^{*}\right)-n x_{1}^{*}\left(x_{3}-x_{3}^{*}\right)}{\left(1+m x_{1}+n x_{3}\right)\left(1+m x_{1}^{*}+n x_{3}^{*}\right)} \\
& \times\left(x_{3}-x_{3}^{*}\right)+k_{2} a_{23} \frac{\alpha\left(x_{2}-x_{2}^{*}\right)\left(x_{3}-x_{3}^{*}\right)}{\left(\alpha+\beta x_{2}\right)\left(\alpha+\beta x_{2}^{*}\right)} \\
& \left.+\frac{\sigma_{3}^{2} x_{3}^{*}}{2}\left(x_{3}-x_{3}^{*}\right)^{2}\right\} \\
& =\left(x_{1}-x_{1}^{*}\right)\left(x_{2}-x_{2}^{*}\right) \\
& -\frac{x_{2}}{x_{1}}\left(x_{1}-x_{1}^{*}\right)^{2}-\frac{a_{11} x_{1}^{*}}{r}\left(x_{1}-x_{1}^{*}\right)^{2} \\
& +\frac{\sigma_{1}^{2}\left(x_{1}^{*}\right)^{2}}{2 r}\left(x_{1}-x_{1}^{*}\right)^{2}
\end{aligned}
$$




$$
\begin{aligned}
& +\frac{m a_{13} x_{1}^{*} x_{3}^{*}}{r\left(1+m x_{1}+n x_{3}\right)\left(1+m x_{1}^{*}+n x_{3}^{*}\right)} \\
& \times\left(x_{1}-x_{1}^{*}\right)^{2}+\left(x_{1}-x_{1}^{*}\right)\left(x_{2}-x_{2}^{*}\right)-\frac{x_{1}}{x_{2}}\left(x_{2}-x_{2}^{*}\right)^{2} \\
& -\frac{a_{22} x_{2}^{*}}{b}\left(x_{2}-x_{2}^{*}\right)^{2} \\
& +\frac{\sigma_{2}^{2}\left(x_{2}^{*}\right)^{2}}{2 b}\left(x_{2}-x_{2}^{*}\right)^{2}+\frac{a_{23} \beta x_{2}^{*} x_{3}^{*}}{b\left(\alpha+\beta x_{2}\right)\left(\alpha+\beta x_{2}^{*}\right)} \\
& \times\left(x_{2}-x_{2}^{*}\right)^{2}-\frac{x_{1}^{*}\left(1+m x_{1}^{*}\right)}{r k_{1}\left(1+n x_{3}^{*}\right)} \\
& \times\left(a_{33}-\frac{\sigma_{3}^{2} x_{3}^{*}}{2}\right)\left(x_{3}-x_{3}^{*}\right)^{2} \\
& -\frac{n a_{13}\left(x_{1}^{*}\right)^{2}\left(1+m x_{1}^{*}\right)\left(x_{3}-x_{3}^{*}\right)^{2}}{r\left(1+n x_{3}^{*}\right)\left(1+m x_{1}+n x_{3}\right)\left(1+m x_{1}^{*}+n x_{3}^{*}\right)} \\
& -\frac{a_{23} x_{2}^{*}\left(x_{2}-x_{2}^{*}\right)\left(x_{3}-x_{3}^{*}\right)}{b\left(\alpha+\beta x_{2}\right)} \\
& +\frac{\alpha k_{2} a_{23} x_{1}^{*}\left(1+m x_{1}^{*}\right)\left(x_{2}-x_{2}^{*}\right)\left(x_{3}-x_{3}^{*}\right)}{r k_{1}\left(1+n x_{3}^{*}\right)\left(\alpha+\beta x_{2}\right)\left(\alpha+\beta x_{2}^{*}\right)} \\
& \leq-\left[\sqrt{\frac{x_{2}}{x_{1}}}\left(x_{1}-x_{1}^{*}\right)-\sqrt{\frac{x_{1}}{x_{2}}}\left(x_{2}-x_{2}^{*}\right)\right]^{2} \\
& -\frac{x_{1}^{*}}{r}\left[a_{11}-\frac{m a_{13} x_{3}^{*}}{1+m x_{1}^{*}+n x_{3}^{*}}-\frac{\sigma_{1}^{2} x_{1}^{*}}{2}\right] \\
& \times\left(x_{1}-x_{1}^{*}\right)^{2} \\
& -\frac{x_{2}^{*}}{b}\left[a_{22}-\frac{a_{23} \beta x_{3}^{*}}{\alpha\left(\alpha+\beta x_{2}^{*}\right)}-\frac{\sigma_{2}^{2} x_{2}^{*}}{2}\right] \\
& \times\left(x_{2}-x_{2}^{*}\right)^{2}-\frac{x_{1}^{*}\left(1+m x_{1}^{*}\right)}{r k_{1}\left(1+n x_{3}^{*}\right)} \\
& \times\left[a_{33}-\frac{\sigma_{3}^{2} x_{3}^{*}}{2}\right]\left(x_{3}-x_{3}^{*}\right)^{2} \\
& +\frac{a_{23} x_{2}^{*}}{b \alpha}\left|x_{2}-x_{2}^{*}\right|\left|x_{3}-x_{3}^{*}\right| \\
& +\frac{k_{2} a_{23} x_{1}^{*}\left(1+m x_{1}^{*}\right)}{r k_{1}\left(1+n x_{3}^{*}\right)\left(\alpha+\beta x_{2}^{*}\right)} \\
& \times\left|x_{2}-x_{2}^{*}\right|\left|x_{3}-x_{3}^{*}\right| \\
& \leq-\frac{x_{1}^{*}}{r}\left[a_{11}-\frac{m a_{13} x_{3}^{*}}{1+m x_{1}^{*}+n x_{3}^{*}}-\frac{\sigma_{1}^{2} x_{1}^{*}}{2}\right] \\
& \times\left(x_{1}-x_{1}^{*}\right)^{2}-\frac{x_{2}^{*}}{b}\left[a_{22}-\frac{a_{23} \beta x_{3}^{*}}{\alpha\left(\alpha+\beta x_{2}^{*}\right)}-\frac{\sigma_{2}^{2} x_{2}^{*}}{2}\right]
\end{aligned}
$$
have

$$
\begin{aligned}
& \times\left(x_{2}-x_{2}^{*}\right)^{2}-\frac{x_{1}^{*}\left(1+m x_{1}^{*}\right)}{r k_{1}\left(1+n x_{3}^{*}\right)}\left[a_{22}-\frac{\sigma_{3}^{2} x_{3}^{*}}{2}\right] \\
& \times\left(x_{3}-x_{3}^{*}\right)^{2}+\frac{a_{23} x_{2}^{*}}{b \alpha}\left|x_{2}-x_{2}^{*}\right|\left|x_{3}-x_{3}^{*}\right| \\
& +\frac{k_{2} a_{23} x_{1}^{*}\left(1+m x_{1}^{*}\right)}{r k_{1}\left(1+n x_{3}^{*}\right)\left(\alpha+\beta x_{2}^{*}\right)}\left|x_{2}-x_{2}^{*}\right|\left|x_{3}-x_{3}^{*}\right| .
\end{aligned}
$$

Let $\left|Z-Z^{*}\right|=\left(\left|x_{1}-x_{1}^{*}\right|,\left|x_{2}-x_{2}^{*}\right|,\left|x_{3}-x_{3}^{*}\right|\right)^{T}$; then we

$$
L V(x) \leq \frac{1}{2}\left|Z-Z^{*}\right|^{T}\left(\begin{array}{ccc}
2 A & 0 & 0 \\
0 & 2 B & D \\
0 & D & 2 C
\end{array}\right)\left|Z-Z^{*}\right|
$$

Clearly, the conditions of Theorem 2 and the above inequality denote $L V(x)<0$ along all trajectories in $R_{+}^{3}$ except $\left(x_{1}^{*}, x_{2}^{*}, x_{3}^{*}\right)$. Then, we get the desired assertion immediately.

For deterministic system (5), we have that the following theorem holds.

Theorem 3. If

$$
\begin{gathered}
a_{11}-\frac{m a_{13} x_{3}^{*}}{1+m x_{1}^{*}+n x_{3}^{*}}>0, \\
a_{22}-\frac{a_{23} \beta x_{3}^{*}}{\alpha\left(\alpha+\beta x_{2}^{*}\right)}>0, \\
\frac{4 a_{33} x_{2}^{*} x_{1}^{*}\left(1+m x_{1}^{*}\right)}{b r k_{1}\left(1+n x_{3}^{*}\right)}\left[a_{22}-\frac{a_{23} \beta x_{3}^{*}}{\alpha\left(\alpha+\beta x_{2}^{*}\right)}\right] \\
-\left[\frac{a_{23} x_{2}^{*}}{b \alpha}+\frac{k_{2} a_{23} x_{1}^{*}\left(1+m x_{1}^{*}\right)}{r k_{1}\left(1+n x_{3}^{*}\right)\left(\alpha+\beta x_{2}^{*}\right)}\right]^{2}>0,
\end{gathered}
$$

then the equilibrium position $\left(x_{1}^{*}, x_{2}^{*}, x_{3}^{*}\right)$ of system (5) is globally asymptotically stable.

Remark 4. From Theorems 2 and 3, we can see that if the positive equilibrium of the deterministic system is globally stable and the noise perturbation is not very large, then the stochastic system will keep the nice property.

\section{Numerical Simulations}

In this section, we will utilize the Milstein method mentioned in Higham [31] to consolidate the analytical findings. 


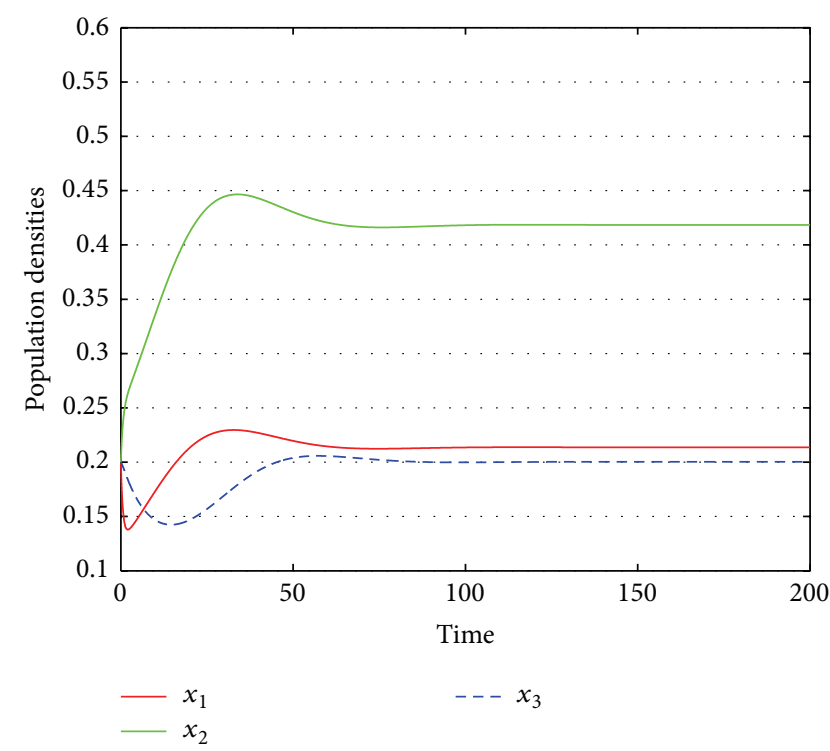

(a)
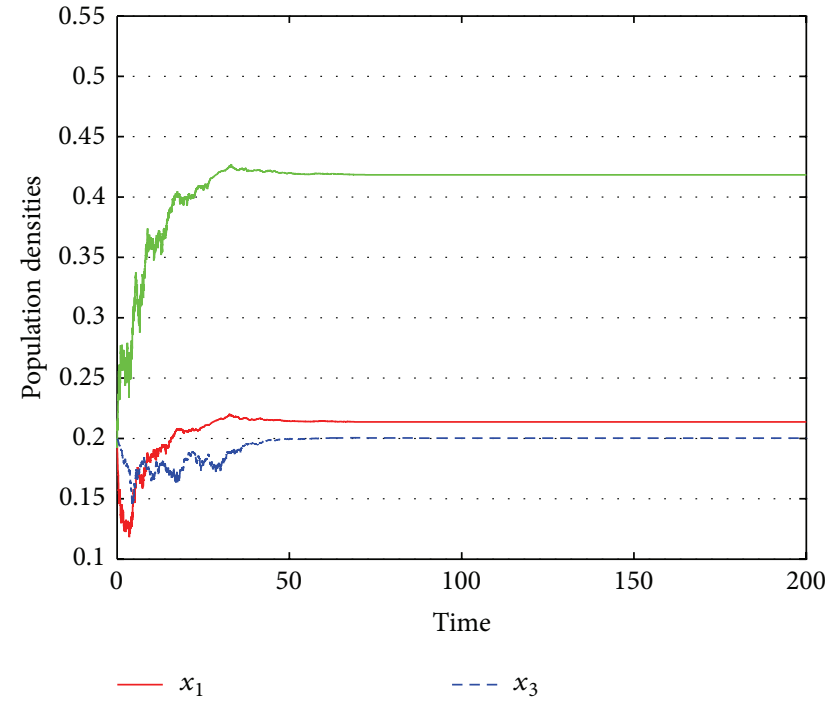

(b)

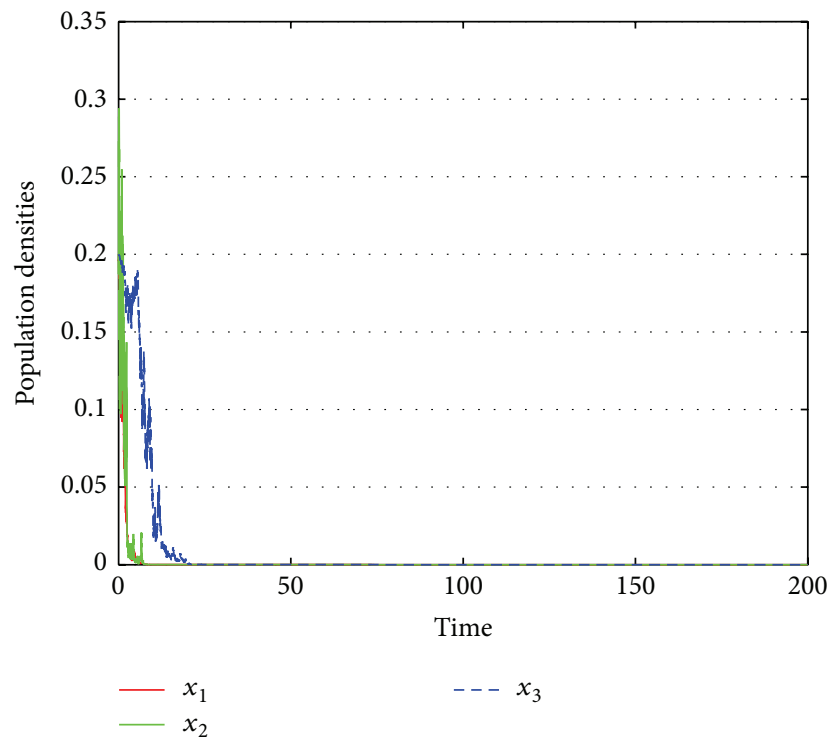

(c)

FIGURE 1: Solution of system (3) with initial conditions $x_{1}(0)=x_{2}(0)=x_{3}(0)=0.2$ and $r=0.6, d_{1}=0.2, a_{11}=0.3, b=0.8, a_{13}=0.6$, $d_{2}=0.3, a_{22}=0.2, a_{23}=0.3, d_{3}=0.1, k_{1}=5 / 6, k_{2}=0.8, a_{33}=0.2, \Delta t=0.01, m=0.3, n=0.1, \alpha=2$, and $\beta=1$. (a) $\sigma_{1}=\sigma_{2}=\sigma_{3}=0$. (b) $\sigma_{1}^{2}=1.9, \sigma_{2}^{2}=0.6$, and $\sigma_{3}^{2}=1.9$. (c) $\sigma_{1}^{2}=20, \sigma_{2}^{2}=20$, and $\sigma_{3}^{2}=20$.

Here, we consider the discretization equations of model (3) as follows:

$$
\begin{aligned}
& x_{1}(k+1) \\
& =x_{1}(k)+\left[r x_{2}(k)-d_{1} x_{1}(k)-a_{11} x_{1}^{2}(k)\right. \\
& \left.\quad-b x_{1}(k)-\frac{a_{13} x_{3}(k)}{1+m x_{1}(k)+n x_{3}(k)}\right] \Delta t \\
& -\sigma_{1} x_{1}(k)\left(x_{1}(k)-x_{1}^{*}\right) \sqrt{\Delta t} \xi_{k}+\frac{\sigma_{1}^{2}}{2} x_{1}(k)
\end{aligned}
$$

$$
\begin{gathered}
\quad \times\left(x_{1}(k)-x_{1}^{*}\right)^{2}\left[\xi_{k}^{2}-1\right] \Delta t, \\
x_{2}(k+1) \\
=x_{2}(k)+\left[b x_{1}(k)-d_{2} x_{2}(k)\right. \\
\left.\quad-a_{22} x_{2}^{2}(k)-\frac{a_{23} x_{3}(k)}{\alpha+\beta x_{2}(k)}\right] \Delta t \\
-\sigma_{2} x_{2}(k)\left(x_{2}(k)-x_{2}^{*}\right) \sqrt{\Delta t} \eta_{k} \\
+\frac{\sigma_{2}^{2}}{2} x_{2}(k)\left(x_{2}(k)-x_{2}^{*}\right)^{2}\left[\eta_{k}^{2}-1\right] \Delta t,
\end{gathered}
$$




$$
\begin{gathered}
x_{3}(k+1) \\
=x_{3}(k)+\left[-d_{3} x_{3}(k)+\frac{k_{1} a_{13} x_{1}(k)}{1+m x_{1}(k)+n x_{3}(k)}\right. \\
\left.\quad+\frac{k_{2} a_{23} x_{2}(k)}{\alpha+\beta x_{2}(k)}-a_{33} x_{3}^{2}(k)\right] \Delta t \\
-\sigma_{3} x_{3}(k)\left(x_{3}(k)-x_{3}^{*}\right) \sqrt{\Delta t} \zeta_{k} \\
+\frac{\sigma_{3}^{2}}{x_{3}}(k)\left(x_{3}(k)-x_{3}^{*}\right)^{2}\left[\zeta_{k}^{2}-1\right] \Delta t
\end{gathered}
$$

where $\xi_{k}, \eta_{k}$, and $\zeta_{k},(k=1,2, \ldots)$ are the Gaussian random variables which follow $N(0,1)$.

Set $r=0.6, d_{1}=0.2, a_{11}=0.3, b=0.8, a_{13}=0.6$, $d_{2}=0.3, a_{32}=0.2, a_{23}=0.3, d_{3}=0.1, k_{1}=5 / 6, k_{2}=0.8$, $a_{33}=0.2, \alpha=2, \beta=1, m=0.3, n=0.1$, and $\Delta t=0.01$. From Figure 1, we can get that the equilibrium is $x_{1}=0.2137$, $x_{2}=0.4184$, and $x_{3}=0.2003$. The only difference between the conditions of Figures 1(a), 1(b), and 1(c) is the values of $\sigma_{1}, \sigma_{2}$, and $\sigma_{3}$. In Figure $1(\mathrm{a})$, we suppose that $\sigma_{1}=\sigma_{2}=$ $\sigma_{3}=0$. By Theorem 3 , the equilibrium point $\left(x_{1}^{*}, x_{2}^{*}, x_{3}^{*}\right)$ of deterministic system (5) is globally asymptotically stable. Figure 1(a) confirms this. In Figure 1(b), we choose $\sigma_{1}^{2}=1.9$, $\sigma_{2}^{2}=0.6$, and $\sigma_{3}^{2}=1.9$. From Theorem 2 , we can easily get that the equilibrium position $\left(x_{1}^{*}, x_{2}^{*}, x_{3}^{*}\right)$ of stochastic system (3) is globally asymptotically stable. By Figure 1(c), we can see that if we choose $\sigma_{1}^{2}=20, \sigma_{2}^{2}=20$, and $\sigma_{3}^{2}=20$, these values violate conditions (19); all the species will die out. That is to say, if the conditions of Theorem 2 are not satisfied, the positive equilibrium point $\left(x_{1}^{*}, x_{2}^{*}, x_{3}^{*}\right)$ may be no longer globally asymptotically stable.

\section{Conclusions}

In this paper, we investigated two stage-structured predatorprey systems: deterministic one and stochastic one. In the models, we suppose that both immature prey and mature prey are consumed by predator. The model is more realistic and complicated than the one in which only the immature prey or mature prey is preyed on by predator. For each system, we established the sufficient conditions for global asymptotic stability. From the results and simulation figures, we can see that if the positive equilibrium position of the corresponding deterministic model is globally stable and the noise is sufficiently small, then the stochastic system will preserve the nice property. The result is useful and important for ecological balance. Up to our knowledge, the present work is the first attempt to study such stochastic model with stage structure on prey.

\section{Conflict of Interests}

The authors declare that there is no conflict of interests regarding the publication of this paper.

\section{Acknowledgments}

The authors would like to thank the referee and editor for their valuable comments and suggestions that greatly improved the presentation of this paper. This work was supported by the Program for New Century Excellent Talents in University (NCET-10-0097), the NSFC Tianyuan Foundation (Grant no. 11226256), and the Zhejiang Provincial Natural Science Foundation of China (Grant no. LY13A010010).

\section{References}

[1] S. Chen and J. Shi, "Global stability in a diffusive Holling-Tanner predator-prey model," Applied Mathematics Letters, vol. 25, no. 3, pp. 614-618, 2012.

[2] F. D. Chen, Y. M. Chen, and J. L. Shi, "Stability of the boundary solution of a nonautonomous predator-prey system with the Beddington-DeAngelis functional response," Journal of Mathematical Analysis and Applications, vol. 344, pp. 1057-1067, 2008 .

[3] T. K. Kar and A. Ghorai, "Dynamic behaviour of a delayed predator-prey model with harvesting," Applied Mathematics and Computation, vol. 217, no. 22, pp. 9085-9104, 2011.

[4] A. Ruiz-Herrera, "Chaos in predator-prey systems with/without impulsive effect," Nonlinear Analysis: Real World Applications, vol. 13, no. 2, pp. 977-986, 2012.

[5] B. Liu, Z. Teng, and L. Chen, "Analysis of a predator-prey model with Holling II functional response concerning impulsive control strategy," Journal of Computational and Applied Mathematics, vol. 193, no. 1, pp. 347-362, 2006.

[6] S. Devi, "Effects of prey refuge on a ratio-dependent predatorprey model with stage-structure of prey population," Applied Mathematical Modelling, vol. 37, no. 6, pp. 4337-4349, 2013.

[7] Y. Shao and B. Dai, "The dynamics of an impulsive delay predator-prey model with stage structure and Beddington-type functional response," Nonlinear Analysis: Real World Applications, vol. 11, no. 5, pp. 3567-3576, 2010.

[8] C.-Y. Huang, Y.-J. Li, and H.-F. Huo, "The dynamics of a stage-structured predator-prey system with impulsive effect and Holling mass defence," Applied Mathematical Modelling, vol. 36, no. 1, pp. 87-96, 2012.

[9] X. Zhang, L. Chen, and A. U. Neumann, "The stage-structured predator-prey model and optimal harvesting policy," Mathematical Biosciences, vol. 168, no. 2, pp. 201-210, 2000.

[10] S. Wang, "Research on the suitable living environment of Rana Temporaria Chensinensis Larva," Chinese Journal of Zoology, vol. 1, pp. 32-38, 1997.

[11] T. Saha and C. Chakrabarti, "Stochastic analysis of preypredator model with stage structure for prey," Journal of Applied Mathematics and Computing, vol. 35, no. 1-2, pp. 195-209, 2011.

[12] E. Beretta, V. Kolmanovskii, and L. Shaikhet, "Stability of epidemic model with time delays influenced by stochastic perturbations," Mathematics and Computers in Simulation, vol. 45, no. 3-4, pp. 269-277, 1998, Delay systems (Lille, 1996).

[13] L. Shaikhet, "Stability of predator-prey model with aftereffect by stochastic perturbation," Stability and Control, vol. 1, no. 1, pp. 3-13, 1998.

[14] W. G. Aiello and H. I. Freedman, "A time-delay model of singlespecies growth with stage structure," Mathematical Biosciences, vol. 101, no. 2, pp. 139-153, 1990. 
[15] T. C. Gard, "Persistence in stochastic food web models," Bulletin of Mathematical Biology, vol. 46, no. 3, pp. 357-370, 1984.

[16] T. C. Gard, "Stability for multispecies population models in random environments," Nonlinear Analysis: Theory, Methods \& Applications, vol. 10, no. 12, pp. 1411-1419, 1986.

[17] R. M. May, Stability and Complexity in Model Ecosystems, Princeton University Press, Princeton, NJ, USA, 2001.

[18] J. R. Beddington and R. M. May, "Harvesting natural populations in a randomly fluctuating environment," Science, vol. 197, pp. 463-465, 1977.

[19] X. Mao, "Stochastic stabilization and destabilization," Systems \& Control Letters, vol. 23, no. 4, pp. 279-290, 1994.

[20] X. Mao, C. Yuan, and J. Zou, "Stochastic differential delay equations of population dynamics," Journal of Mathematical Analysis and Applications, vol. 304, no. 1, pp. 296-320, 2005.

[21] Q. Luo and X. Mao, "Stochastic population dynamics under regime switching," Journal of Mathematical Analysis and Applications, vol. 334, no. 1, pp. 69-84, 2007.

[22] R. Rudnicki and K. Pichór, "Influence of stochastic perturbation on prey-predator systems," Mathematical Biosciences, vol. 206, no. 1, pp. 108-119, 2007.

[23] X. Li and X. Mao, "Population dynamical behavior of nonautonomous Lotka-Volterra competitive system with random perturbation," Discrete and Continuous Dynamical Systems A, vol. 24, no. 2, pp. 523-545, 2009.

[24] M. Liu and K. Wang, "Survival analysis of stochastic singlespecies population models in polluted environments," Ecological Modelling, vol. 220, pp. 1347-1357, 2009.

[25] M. Vasilova, "Asymptotic behavior of a stochastic Gilpin-Ayala predator-prey system with time-dependent delay," Mathematical and Computer Modelling, vol. 57, no. 3-4, pp. 764-781, 2013.

[26] M. Liu and K. Wang, "Global stability of stage-structured predator-prey models with Beddington-DeAngelis functional response," Communications in Nonlinear Science and Numerical Simulation, vol. 16, no. 9, pp. 3792-3797, 2011.

[27] L. Arnold, Stochastic Differential Equations: Theory and Applications, Wiley-Interscience, New York, NY, USA, 1972.

[28] A. Friedman, Stochastic Differential Equations and Applications, vol. 2, Academic Press, New York, NY, USA, 1976.

[29] I. Karatzas and S. E. Shreve, Brownian Motion and Stochastic Calculus, vol. 113, Springer, New York, MY, USA, 2nd edition, 1991.

[30] X. Mao, G. Marion, and E. Renshaw, "Environmental Brownian noise suppresses explosions in population dynamics," Stochastic Processes and Their Applications, vol. 97, no. 1, pp. 95-110, 2002.

[31] D. J. Higham, "An algorithmic introduction to numerical simulation of stochastic differential equations," SIAM Review, vol. 43, no. 3, pp. 525-546, 2001. 


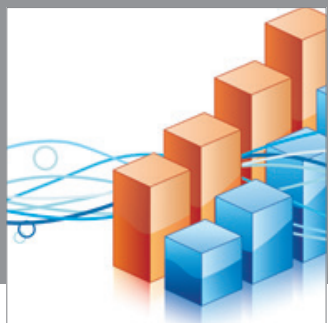

Advances in

Operations Research

mansans

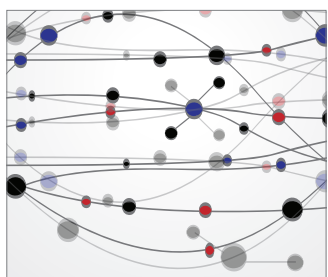

The Scientific World Journal
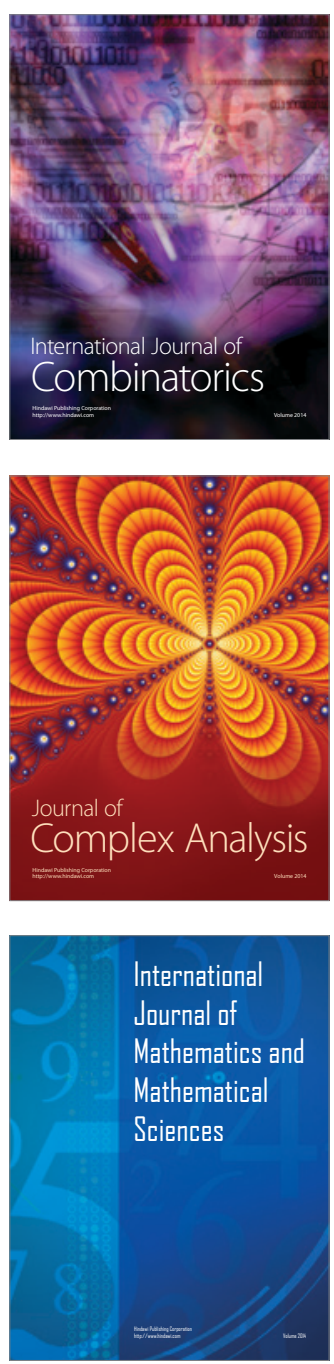
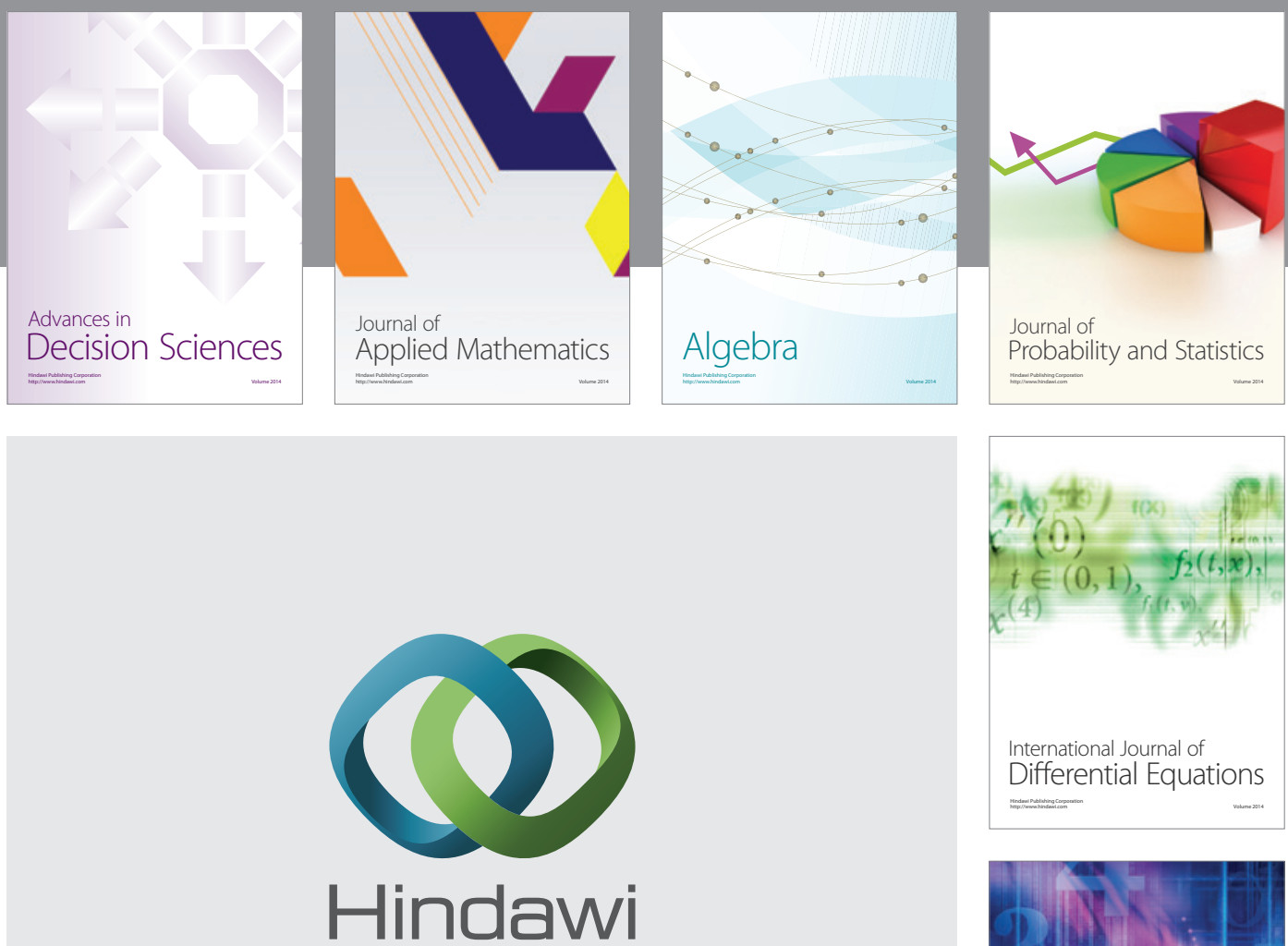

Submit your manuscripts at http://www.hindawi.com
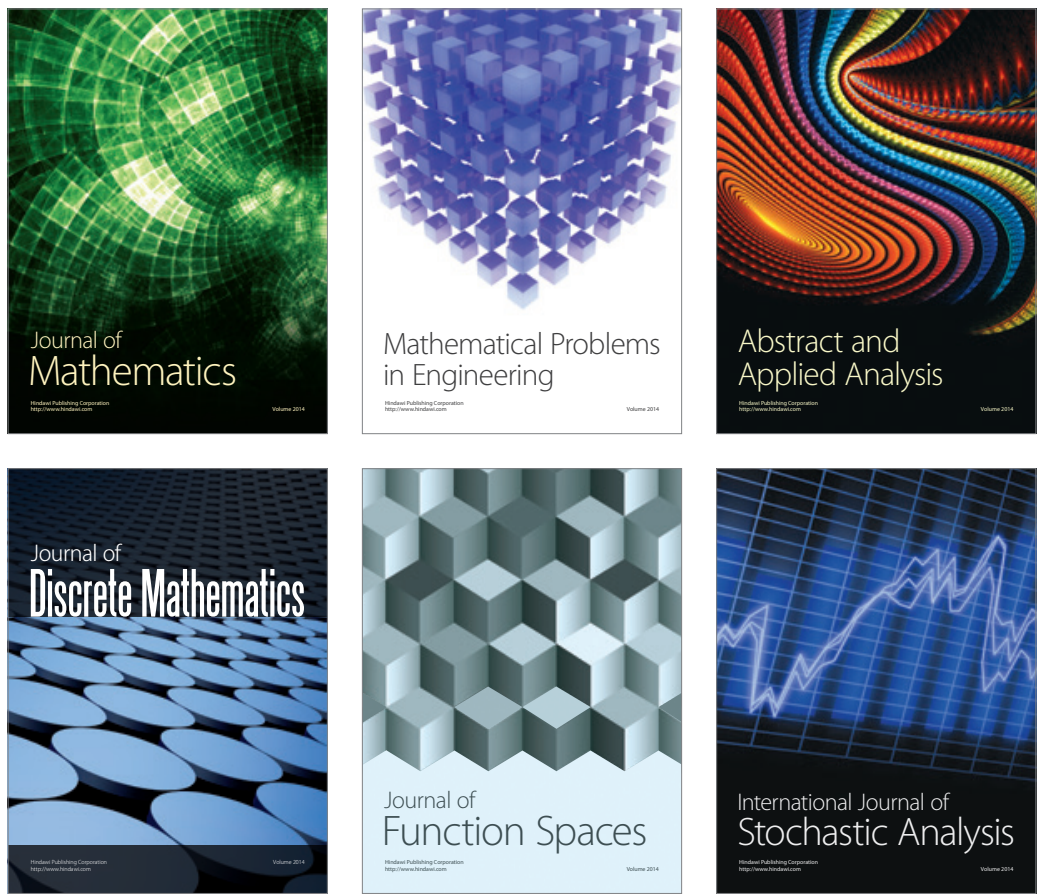

Journal of

Function Spaces

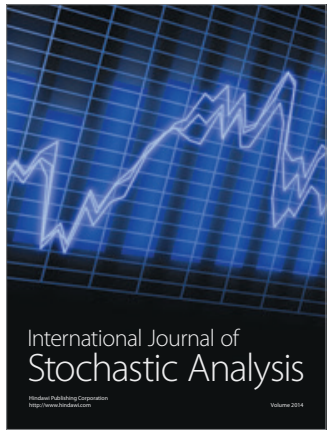

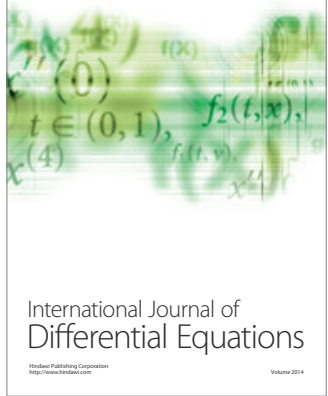
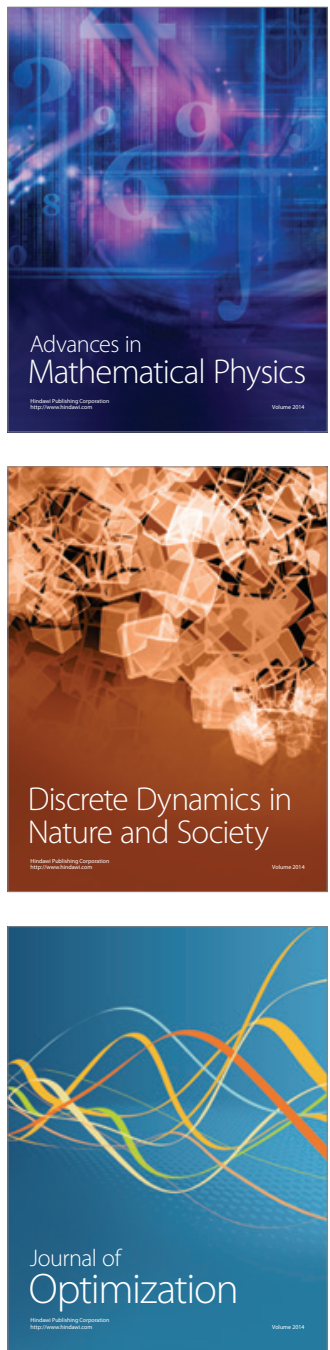